\title{
Gender-Dependent Clinical Outcome and Other Predictors of In-Hospital Mortality Following Out-Of-Center Extracorporeal Membrane Oxygenation and Extracorporeal Life Support: A Single Center Experience
}

\author{
Florian Rückert, ${ }^{1}$ Christoph Raspé, ${ }^{2}$ Giuseppe D’Ancona, ${ }^{3}$ Huesyin Ince,,${ }^{3,4}$ Efstratios I. Charitos, ${ }^{5}$ Alper Öner, ${ }^{4}$ \\ Peter Donndorf, ${ }^{6}$ Hasan Bushnaq ${ }^{6}$ \\ ${ }^{1}$ Department of Anesthesiology and Operative Intensive Care Medicine, Ernst von Bergmann Clinic, Potsdam, Germany; \\ ${ }^{2}$ Department of Anesthesiology, Operative Intensive Care Medicine and Pain Therapy, Vivantes Auguste-Viktoria- and Wenckebach- \\ Clinic, Berlin, Germany; ${ }^{3}$ Department of Cardiology, Vivantes Klinikum im Friedrichshain und am Urban, Berlin, Germany; \\ ${ }^{4}$ Department of Cardiology, Rostock University Medical Center, Rostock, Germany; ${ }^{5}$ Department of Cardiac Surgery, Heart Center \\ Bonn, University Hospital Bonn, Germany; ${ }^{6}$ Department of Cardiac Surgery, University Hospital Rostock, Germany
}

\section{ABSTRACT}

Background: Out-of-center extracorporeal membrane oxygenation (ECMO) and extracorporeal life support (ECLS) implantation for the treatment of acute cardiorespiratory failure with subsequent transport to a tertiary care center has been introduced successfully into the medical practice. However, due to the very specific and resource intensive nature of this therapeutic concept, it seems important to generate algorithms for adequate patient selection. The aim of our study was to analyze the impact of patients' gender on early clinical outcome in this specific therapeutic scenario.

Methods: Ninety-seven consecutive patients treated by out-of-center ECMO/ECLS implantation and subsequent transport and treatment in our tertiary care cardiovascular center within the Hallesche Extracorporeal Life Support Program (HELP) retrospectively were analyzed, regarding the impact of patients' gender on early clinical outcome.

Results: Mechanical circulatory support successfully was weaned in two-thirds of the male patients. This result was achieved in only one-third of the female patients $(59.4 \%$ in male vs. $33.3 \%$ in female, $P=.0267)$. Overall survival significantly was higher in the male group $(62.5 \%$ in male versus $30.3 \%$ in female, $P=$ $.0052)$. In uni- and multivariate logistic regression analysis, female gender was an independent predictor of in-hospital mortality (uni: OR:3.833, CI:1.597-9.745, $P=.0034$; multi: OR:3.477, CI:1.146-11.494, $P=.0322)$. Worse outcome also was associated with following independent predictors, age, SOFA score, lactate and ventilation time pre-ECMO/ECLS implantation.

Conclusion: The current study demonstrates a worse early survival for women, following emergent out-of-

Received April 2, 2020; accepted April 6, 2020.

Correspondence: Florian Rückert, Department of Anesthesiology, Ernst von Bergmann Clinic, Potsdam, Germany; (e-mail: florian.rueckert@klinikumevb.de) center ECMO/ECLS implantation and subsequent transport and treatment in our tertiary care cardiovascular center. Gender should be included in patient selection algorithms while basic research approaches are needed to better understand the mechanisms underlying these genderspecific outcome disparities.

\section{INTRODUCTION}

Acute cardiorespiratory failure remains a life-threatening complication. Especially in critically-ill patients, immediate mechanical circulatory support often is the only therapeutic option to avoid permanent end-organ damage and consequent fatal outcome [Chen 2008; Sayer 2012]. However, the immediate

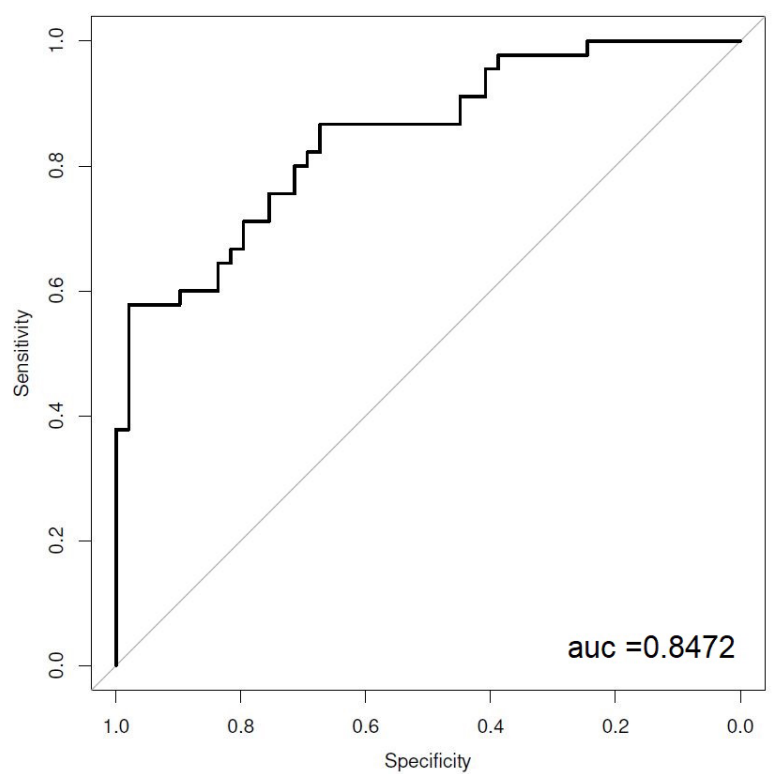

ROC-analysis of multivariate regression model of in-hospital mortality. $\mathrm{AUC}=$ area under the curve. 
Table 1. Baseline parameters prior ECMO/ECLS implantation

\begin{tabular}{|c|c|c|c|c|c|}
\hline Patient number $(n)$ & 97 & $64(65.98 \%)$ & $33(34.02 \%)$ & & \\
\hline Age (years) & $55.0(45.0-64.0)$ & $53.5(44.8-61.0)$ & $59.0(46.0-70.0)$ & .1620 & n.s. \\
\hline Height $(\mathrm{cm})$ & $175.0(166.0-180.0)$ & $179.5(175.0-184.0)$ & $165.0(165.0-168.0)$ & $<.00001$ & $* * *$ \\
\hline BMI $\left(\mathrm{kg} / \mathrm{m}^{2}\right)$ & $28.2(24.8-33.1)$ & $27.8(24.6-33.1)$ & $29.0(25.7-32.7)$ & .3934 & n.s. \\
\hline Hospital stay prior support (days) & $5.0(2.0-9.0)$ & $5.0(2.0-9.0)$ & $7.0(3.0-11.0)$ & .2494 & n.s. \\
\hline Ventilation time prior support (days) & $2.0(1.0-5.0)$ & $2.0(1.0-5.3)$ & $3.0(1.0-5.0)$ & .4302 & n.s. \\
\hline CPR prior support $(n)$ & $19(19.6 \%)$ & $16(25.0 \%)$ & $3(9.1 \%)$ & 1095 & n.s. \\
\hline SOFA score (pts.) & $14.0(12.0-16.0)$ & $14.0(12.0-16.0)$ & $14.0(12.0-16.3)$ & .9291 & n.s. \\
\hline
\end{tabular}

APACHE II: Acute Physiology And Chronic Health Evaluation II; BMI: body mass index; CPR: cardio-pulmonary resuscitation; SOFA: Sequential Organ Failure Assessment; va: veno-arterial; vv: veno-venous; n.s.: non-significant. $* P<.05 * * P<.01 * * * P<.001$; All data are presented as $\mathrm{n}(\%)$ or median (IQR)

Table 2. Respiratory, hemodynamic and laboratory data prior to ECMO/ECLS implantation

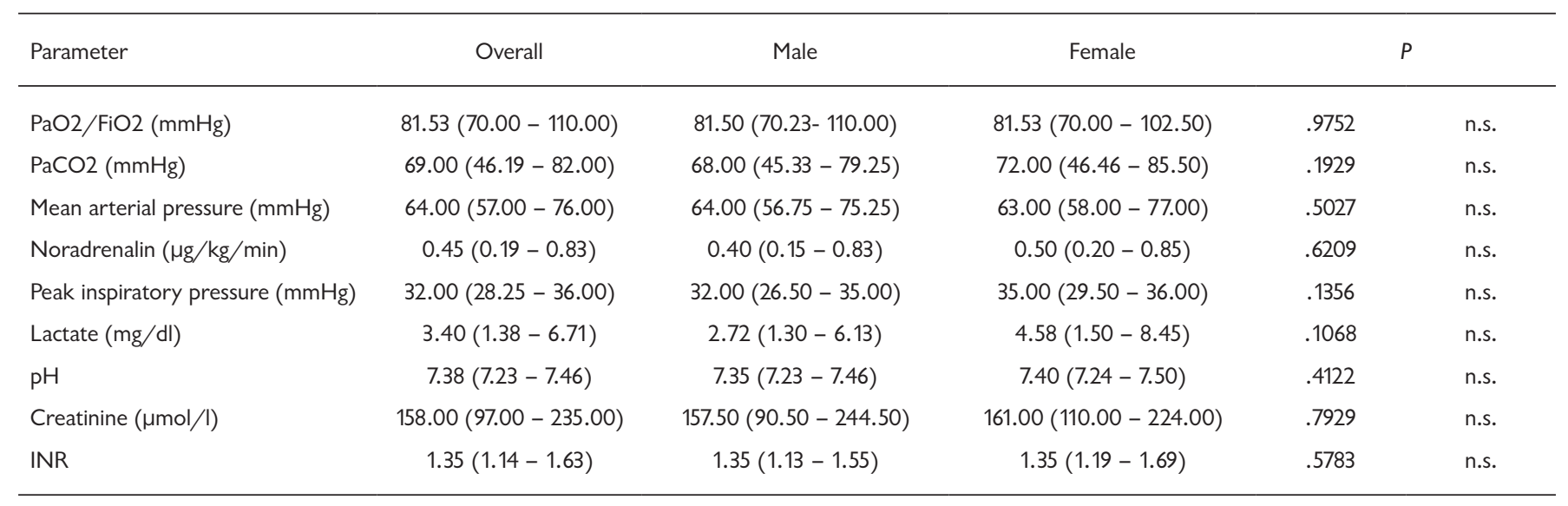

INR: international normalized ratio; n.s.: non-significant; All data are presented as n (\%) or median (IQR)

availability of both extracorporeal membrane oxygenation systems (ECMO, VV-ECMO) for the treatment of respiratory failure and extracorporeal life support systems (ECLS, VA-ECMO) for the treatment of cardiac failure is limited to specialized tertiary care centers, since the use requires multidisciplinary expertise and infrastructure [Beckmann 2011; Philipp 2011; Sayer 2012; Thiagarajan 2009]. As the critical patient's status often prohibits transport to such tertiary care centers, the concept of out-of-center ECMO/ECLS implantation successfully has been introduced into the clinical practice, based on recent advances in mobile mechanical circulatory support technology.

This concept includes the implementation of a specialized and mobile multidisciplinary team on permanent standby for emergent implantation of a ECMO/ECLS system in a remote hospital followed by patient transport to the tertiary care center for subsequent management, thereby increasing the patient's chance of survival [Raspé 2015; Rückert 2017].

However, despite recent advances of this approach toward establishing mobile ECMO/ECLS networks on a regional scale, there still is a lack of adequate patient selection criteria as well as systematic evaluation of early outcome parameters.

In the current retrospective study, we investigated the impact of patients' gender on the early outcome following out-of-center ECMO/ECLS implantation and subsequent transport and therapy to our tertiary care center. 
Table 3. Indication/diagnosis leading to ECMO/ECLS implantation

\begin{tabular}{lc}
\hline Indication/diagnosis & $\mathrm{N}$ \\
\hline ARDS & $73(75.26 \%)$ \\
Pulmonary infection & 36 \\
Aspiration & 10 \\
Sepsis & 7 \\
Other reasons & 10 \\
Unknown & 10 \\
Cardiogenic shock & $24(24.74 \%)$ \\
Myocardial infarction & 14 \\
Dilated cardiomyopathy & 5 \\
Pulmonary embolism & 4 \\
Postoperative cardiomyopathy & 1
\end{tabular}

\section{PATIENTS AND METHODS}

Study design and period: The Hallesche Extracorporeal Life Support Program (HELP) for the treatment of acute respiratory and/or cardiac failure has been introduced in 2009.

We report here 97 consecutive patients treated by ECMO/ ECLS implantation in a remote hospital by our mobile ECMO/ECLS team, since the start of the program in 2009 until 2015. After the implantation and initial hemodynamic stabilization, all patients were transferred to our center for subsequent diagnostic and therapy. In the analyzed cohort, 72 patients required ECMO for primary respiratory failure; 25 patients were treated by ECLS for primary cardiac failure.

Data prospectively were collected and retrospectively analyzed. Patients and/or their legal representatives signed consent to treatment and to the collection of their clinical information for possible future data analysis. The study was approved by our institutional review board. (Ethical Committee of Medical School Martin-Luther-University, Halle-Wittenberg, Germany, working-number 2015-122).

ECMO/ECLS team and logistics: The referring outside hospital contacts our center (Mitteldeutsches Herzzentrum, Halle, Germany) by phone via a central hotline. Relevant medical data (patient demographics, hemodynamic and ventilation parameters, and organ and laboratory values) were transferred via an online platform. A multidisciplinary team on 24/7 standby, consisting of a cardiac surgeon and anesthetist, evaluate the available medical data with our standard protocol. In parallel, the ECMO/ECLS system and required materials are prepared by a perfusion technician on-call. Once the patient has been accepted for treatment, the whole team immediately is dispatched to the referring hospital, where ECMO/ECLS support is instituted as fast as possible.

ECMO/ECLS implantation: Both systems (ECMO and ECLS) are based on a custom-made system named HELPer@ .
The system consists of a centrifugal pump (Centrimag@) St. Jude Medical Thoratec or Revolution 5(), LivaNova) and a membrane oxygenator (Alone ECMO Oxygenator Adult $\odot$, Eurosets). The system is primed with $500 \mathrm{ml}$ of $0.9 \%$ sodium chloride solution. During transport oxygen supply is secured via a 51 oxygen bottle.

Venous cannulation is performed in a percutaneous fashion in all cases via the femoral vein, utilizing a $70 \mathrm{~cm}$ 24-French cannula (Duraflow, Edwards Lifesciences). In case of ECMO support, the second venous cannula is placed via the contralateral femoral vein, thereby placing the outflow cannula in the right atrium and the inflow cannula at the transition of the inferior vena cava and the iliac vein so to avoid blood recirculation. In one patient, percutaneous placement of a second femoral cannula was not feasible so a jugular vein was used for placement of the outflow cannula. The position of the outflow cannula within the right atrium is verified by transthoracic echocardiography and immediately corrected, if necessary.

In the case of ECLS system, a $23 \mathrm{~cm} 17$-French arterial cannula (HLS, Maquet) is placed in a percutaneous fashion in the femoral artery. In addition, all ECLS patients receive another 9 French arterial cannula (Super arrow flex, Arrow) to facilitate antegrade leg perfusion. If there is enough time during implementation of the ECLS system, this cannulation is performed after separate puncture of the femoral artery, otherwise leg perfusion would be monitored through INVOS $^{\mathrm{TM}}$ Somatic Oximetry Adult Sensors (Medtronic). If necessary, additional cannula is placed during ICU stay.

After commencing mechanical circulatory support, stabilization of the hemodynamics and adequate oxygenation, the patient is prepared for transport. Because of the severe hemodynamic or pulmonary situation, the patients were intubated and sedated for transport. (median APACHE-II-Score of 28, first quartile: 25.75 , third quartile: 32.00 ).

During transport, heart rate, invasive arterial blood pressure, and peripheral oxygen saturation continuously are monitored. Peripheral oxygenation and blood pressure are invasively measured at the right forearm in the arteria radialis in order to estimate effective coronary and cerebral perfusion. Mean arterial blood pressure over $60 \mathrm{mmHg}$ and peripheral oxygen saturation over $90 \%$ are the goals of treatment.

For more information about methods and material, please consult previously published articles of this study [Raspé 2015; Rückert 2017].

Statistical methods: Two groups were identified, according to gender. Due to the non-normally distributed data, MannWhitney-U-Test was used for the comparison of quantitative variables between the male and female groups. The tests were two-tailed, and $P$-values of $<.05$ were considered statistically significant. Categorical variables were compared using chi-square test and Fischer-exact test whenever required. Uni- and multivariate linear regression analysis was used to verify gender and other parameters as independent predictors of mortality. Regression modelling estimates are presented as the mean ( $95 \%$ confidence intervals). 
Table 4. Respiratory, hemodynamic, laboratory data post ECMO/ECLS implantation and clinical outcome following ECMO/ECLS implantation

\begin{tabular}{|c|c|c|c|c|c|}
\hline $\mathrm{PaO} 2 / \mathrm{FiO} 2(\mathrm{mmHg})$ & $142.29(101.95-247.51)$ & $158.17(104.57-261.28)$ & $129.25(97.91-201.14)$ & .3450 & n.s. \\
\hline Mean arterial pressure $(\mathrm{mmHg})$ & $80.50(70.50-91.50)$ & $81.00(70.50-89.25)$ & $79.00(69.75-96.50)$ & .8057 & n.s. \\
\hline Noradrenalin $(\mu \mathrm{g} / \mathrm{kg} / \mathrm{min})$ & $0.14(0.04-0.40)$ & $0.13(0.04-0.31)$ & $0.16(0.06-0.50)$ & .4909 & n.s. \\
\hline Gas flow ECMO (I/min) & $5.00(4.00-6.00)$ & $4.50(3.75-6.50)$ & $5.00(4.00-5.25)$ & .7348 & n.s. \\
\hline $\mathrm{FiO} 2$ ventilator $(\%)$ & $73.00(56.00-90.00)$ & $71.00(53.50-90.50)$ & $75.00(59.50-90.00)$ & .6989 & n.s. \\
\hline FiO2 ECMO (\%) & $90.00(80.00-100.00)$ & $100.00(80.00-100.00)$ & $80.00(77.50-100.00)$ & .0594 & n.s. \\
\hline rpm ECMO & $3400.00(2937.75-3900.00)$ & $3325.00(2805.50-3887.50)$ & $3500.00(3175.00-4050.00)$ & .1128 & n.s. \\
\hline Blood flow $(\mathrm{l} / \mathrm{min})$ & $4.20(3.67-4.92)$ & $4.20(3.62-5.00)$ & $4.10(3.73-4.69)$ & .9397 & n.s. \\
\hline Weaning (N, \%) & $49(50.52 \%)$ & $38(59.38 \%)$ & $11(33.33 \%)$ & .0267 & * \\
\hline ICU time (days) & $22.00(10.00-45.00)$ & $26.50(13.00-45.25)$ & $19.00(7.00-35.00)$ & .1374 & n.s. \\
\hline Overall survival (N, \%) & $50(51.55 \%)$ & $40(62.50 \%)$ & $10(30.30 \%)$ & .0052 & $* *$ \\
\hline Time on ECMO/ECLS (days) & $11.00(7.00-17.00)$ & $11.00(8.00-18.00)$ & $12.00(6.00-17.00)$ & .8878 & n.s. \\
\hline
\end{tabular}

ICU: intensive care unit; rpm: rounds per minute; n.s.: non-significant; All data are presented as $\mathrm{n}(\%)$ or median (IQR).

$* P<.05 * * P<.01 * * * P<.001$

\section{RESULTS}

ECMO/ECLS implantation and baseline data: A total 97 patients were treated with an emergent ECMO/ ECLS implantation in an outside hospital. ECMO/ECLS implantation was uneventful in all cases. Of the patients, 72 were treated with an ECMO system, due to respiratory failure; 25 patients received an ECLS system, due to acute cardiac failure. There were 64 male patients and 33 female patients.

There only were only two significant differences between the two gender groups, regarding the baseline medical data (Table 1). Female patients had a smaller body size $(P<.00001)$ and a higher Murray-Score before ECMO/ECLS was implanted $(P=.0096)$. The pre-implantation hemodynamic and respiratory data immediately before ECMO positioning also were similar in the two groups (Table 2).

The most frequent diagnoses leading to ECMO/ECLS implantation are listed in Table 3. Pulmonary infection was the main cause for implantation of ECMO, while acute myocardial infarction (with or without cardiopulmonary resuscitation) was leading cause for implantation of ECLS (Table 3). Post-implant hemodynamics, oxygenation, and organ function: In both groups, ECMO/ECLS implantation resulted in an optimized oxygenation and hemodynamic status. Optimized oxygenation resulted in higher $\mathrm{PaO} 2 / \mathrm{FiO} 2$ ratio in both groups, whereas hemodynamic stabilization resulted in higher mean arterial pressures and reduced doses of noradrenalin. Consequently, re-compensation of organ function was achieved in both groups, represented by a significant drop of serum creatinine compared with pre-implantation values (Table 4).

After 72 hours of ECMO/ECLS therapy, significant higher values of $\mathrm{PaO} 2 / \mathrm{FiO} 2$ and mean arterial pressure $(P<.001$, $P<.001)$ and significant lower values of Ppeak, Norepinephrine and $\mathrm{PaCO} 2(P<.001, P=.013, P<.001$, respectively $)$ were achieved.

The main functional parameters of the ECMO/ ECLS systems, such as blood flow, rates per minute and Oxygenator $\mathrm{FiO} 2$ did not significantly differ between the two groups (Table 4). Only peak inspiratory pressure after 72 hours of ECMO/ECLS significantly differs between male and female patients $(P=.0079)$.

Clinical outcome: Duration of the ICU stay, respirator times and mechanical circulatory support times did not significantly differ between the two groups. However, there was a significant difference, regarding the percentage of patients successfully weaned from the ECMO/ECLS system and likewise regarding the overall survival. While mechanical circulatory support successfully was weaned in two-thirds of the male patients, this result was achieved in only one-third of the female patients $(59.4 \%$ in male versus $33.3 \%$ in female, 
Table 5. Thromboembolic and bleeding complications $(\mathrm{N})$ during the study period, consumption of blood products and renal replacement therapy

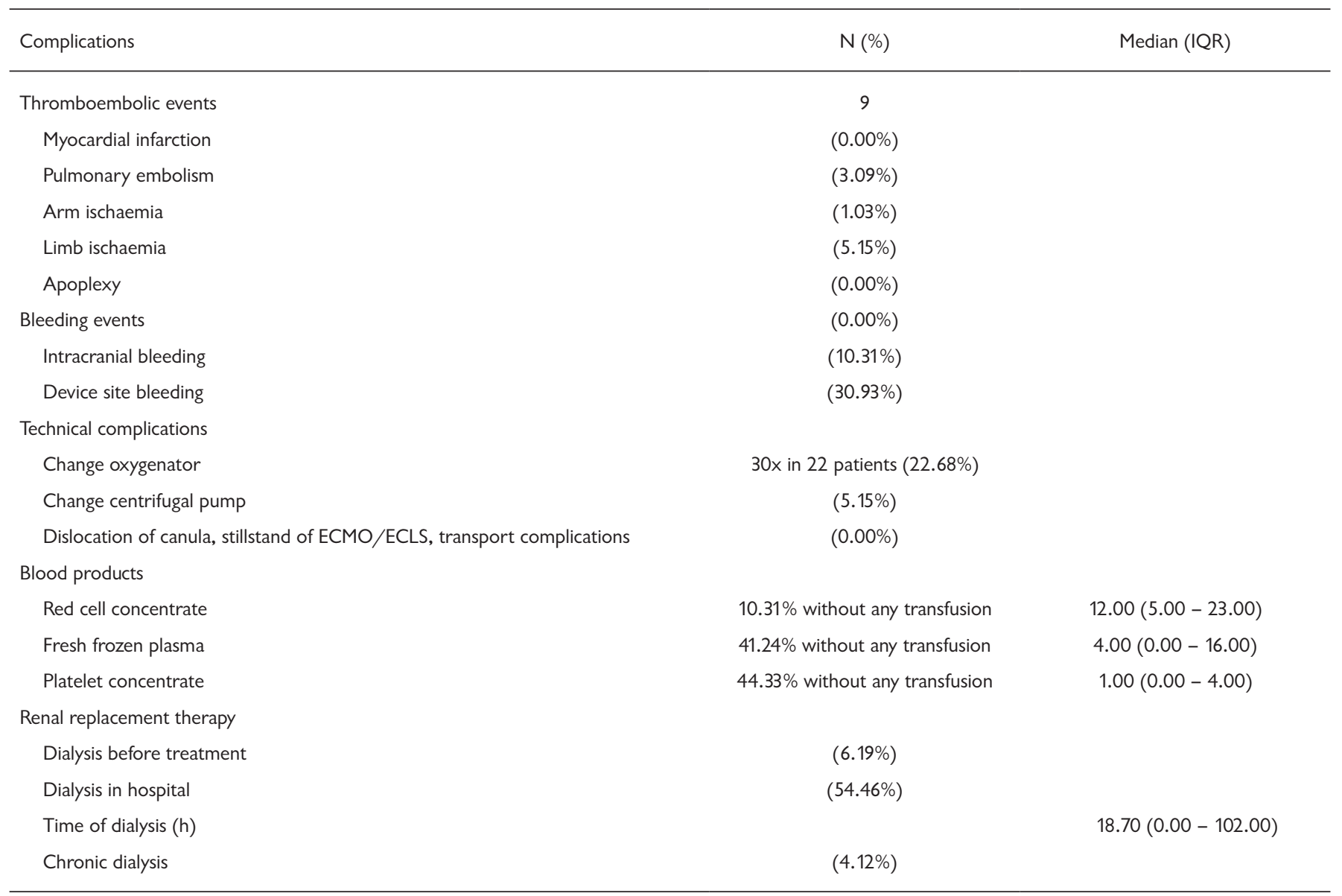

All data are presented as $\mathrm{N}(\%)$ or Median (IQR)

$P=.0267)$. Similarly, overall survival significantly was higher in the male group $(62.5 \%$ in male versus $30.3 \%$ in female, $P=.0052)$ (Table 4). Complications, transfusion management and renal replacement therapy during the whole hospital stay are shown in Table 5.

To identify predictors of in-hospital mortality, a univariate logistic regression analysis was performed (gender, age, pre-ECMO/ECLS hospital and ventilation days, SOFA score, APACHE II score, Murray score, $\mathrm{PaO} 2 / \mathrm{FiO} 2, \mathrm{PaCO}$, lactate, $\mathrm{pH}$, PEEP, INR, dialysis, cancer disease). With the significant factors of univariate logistic regression, we performed a multivariate logistic regression analysis and were able to identify female gender, age, pre-ECMO/ECLS ventilation days, SOFA score and lactate as independent predictors for in-hospital mortality (Table 6). In multivariate analysis, female gender was the strongest independent predictor of inhospital mortality. (Odds ratio $3.477,95 \%$ confidence interval 1.146-11.494, $P=.032$ for multivariate regression).

To test the created model, we also performed a ROCanalysis (receiver operating characteristic). The area under the curve was 0.8472 (Figure).

\section{DISCUSSION}

Recent clinical evidence underlining the general efficacy of ECMO/ECLS systems in specific acute clinical settings has further supported the implementation of concepts for out-of-center ECMO/ECLS implantation [Raspé 2015; Rückert 2017].

For example, both the results from the CESAR trial [Peek 2010] as well the reported results among patients with severe H1N1 influenza [Noah 2011] have supported the different ECMO systems as a major therapeutic option in acute respiratory distress syndrome. In case of acute cardiac failure, a recent meta-analysis reported not only improved neurologic outcome and survival in patients treated with ECLS [Khorsandi 2017; Sheu 2010], but also a significant advantage compared with treatment with an intra-aortic balloon pump [Ouweneel 2016].

Due to technical improvements resulting in high compactness of both systems, out-of-center implantation increasingly is feasible in all of the described clinical settings and allows for early onset of the organ support and coverage of the patient transport back to tertiary care centers [Philipp 2011]. Due to the given complexity of this acute therapeutic setting, 
Table 6. Multivariate logistic regression analysis of in-hospital mortality

\begin{tabular}{lcccc}
\hline Factor & Odds Ratio & $95 \%-\mathrm{Cl}$ & $P$ \\
\hline Female gender & 3.477 & $1.146-11.494$ & .0322 & .0077 \\
Age & 1.067 & $1.021-1.122$ & .0322 & .0358 \\
Ventilation pre ECMO/ECLS (day) & 1.162 & $1.023-1.351$ & .0419 & .0509 \\
SOFA Score (point) & 1.207 & $1.019-1.453$ & $1.015-1.356$ & $*$ \\
Lactate (mmol/l) & 1.161 & $1.058-16.792$ & $*$ \\
Cancer disease pre ECMO/ECLS & 3.854 & n.s. \\
\hline
\end{tabular}

ECLS: Extracorporeal Life Support, ECMO: Extracorporeal Membrane Oxygenation, SOFA: Sequential Organ Failure Assessment score; n.s.: non-significant. $* P<.05 * * P<.01 * * * P<.001$

prospective and randomized data, regarding the early survival and clinical outcome following out-of-center ECMO/ECLS implantation, is relatively rare. Current evidence consists mainly of retrospective analyses reporting a 30-day survival of around $30 \%$ in most studies.

However, with the required logistic for out-of-center ECMO/ ECLS implantation set up in and around several tertiary care centers, it seems important to generate adequate algorithms for patient selection and/or risk stratification for this very specific and resource intensive therapeutic concept [Combes 2017]. One main criterion in such algorithms is the patient's gender.

The impact of the patient's gender on early survival, following out-of-center ECMO/ECLS implantation, has not conclusively been analyzed so far, although such data already exists for the setting of long-term mechanical circulatory support. For example, results from the EUROMACS registry report relevant gender differences, regarding outcomes after isolated LVAD implantation with the survival of women on isolated LVAD support described as significantly worse compared with men [Magnussen 2018]. In fact, women and men differed in perioperative hemodynamics, adverse events, and mortality after VAD implantation. A gender-dependent association of pump thrombosis with mortality was seen [Magnussen 2018].

Equivalently, a recent meta-analysis suggests women are at greater risk of significant complications, such as cerebrovascular events and right heart failure, after LVAD implantation [Blumer 2018]. Cifkova et. al described various gender-differences in the context of cardiovascular diseases [Cifkova 2019].

In line with these findings, our results following out-ofcenter ECMO/ECLS implantation and transport to our tertiary care center by our institutional team demonstrate a significantly worse survival for women, despite a lower incidence of pre-implant CPR in female patients, indicating an even more critical pre-implant status of analyzed male patients. Other baseline characteristics prior ECMO/ECLS implantation did not differ significantly between the two groups and an overall early survival of $50 \%$ was comparable to other reports analyzing out-of-center ECMO/ECLS implantation.

The reason for this outcome discrepancy between men and women obviously cannot be answered conclusively in a retrospective study like ours; it will require basic research approaches.

For example, authors investigating gender differences in outcomes after aortic aneurysm surgery suggest investigation of hormonal or molecular explanations for the sex differences in aortic disease [Makrygiannis 2014].

However, although underlying reasons remain to be examined, our current data provide important evidence, regarding the inclusion of patient's gender in algorithms integrating patient selection criteria for out-of-center ECMO/ECLS implantations.

Nevertheless, it must be noted that our results were obtained in only one ECMO/ECLS-center and patients' heterogeneity could influence different results of our study.

In conclusion, in this single center retrospective study, we have demonstrated according to other groups that emergent out-of-center ECMO/ECLS implantation is a feasible therapeutic option for the treatment of acute respiratory and/or cardiac failure when performed by specialized interdisciplinary teams out of a tertiary care center providing dedicated pre- and post-implant logistics. We found significantly worse early survival for women following emergent out-of-center ECMO/ECLS implantation and subsequent transport and treatment in our tertiary care cardiovascular center. Further research is needed to better understand the mechanisms underlying these gender-specific outcome disparities.

\section{ACKNOWLEDGEMENT}

This work was performed in the Department of Heart Surgery and Department of Anesthesiology and Operative Intensive Care Medicine, University Hospital Halle/Saale, University Halle-Wittenberg, Germany" as mentioned in one of the comments in the PDF-file.

\section{REFERENCES}

Raspé C, Rückert F, Metz D, et al. Inter-hospital transfer of ECMOassisted patients with a portable miniaturized ECMO device. Perfusion. 2015 Jan;30(1):52-9. 
Rückert F, Steinke T, Flöther L, et al. Predictors for quality of life of patients with a portable out-of-centre-implanted extracorporeal membrane oxygenation device. Interact Cardiovasc Thorac Surg. 2017 Apr 1;24(4):542-548.

Beckmann A, Benk C, Beyersdorf F, et al. 2011. Position article for the use of extracorporeal life support in adult patients. Eur J Cardiothorac Surg. Sep;40(3):676-80.

Blumer V, Mendirichaga R, Hernandez GA, Zablah G, Chaparro SV. 2018. Sex-Specific Outcome Disparities in Patients Receiving Continuous-Flow Left Ventricular Assist Devices: A Systematic Review and Meta-analysis. ASAIO J. Jul/Aug;64(4):440-449.

Chen YS, Lin JW, Yu HY, et al. 2008. Cardiopulmonary resuscitation with assisted extracorporeal life-support versus conventional cardiopulmonary resuscitation in adults with in-hospital cardiac arrest. Lancet. Aug 16;372(9638):554-61.

Cifkova R, Pitha J, Krajcoviechova A, Kralikova E. 2019. Is the impact of conventional risk factors the same in men and women? Plea for a more gender-specific approach. Int J Cardiol. Jul 1;286:214-219.

Combes A, Pesenti A, Ranieri VM. 2017. Fifty Years of Research in ARDS. Is Extracorporeal Circulation the Future of Acute Respiratory Distress Syndrome Management? Am J Respir Crit Care Med. May 1;195(9):1161-1170.

Khorsandi M, Dougherty S, Bouamra O, et al. 2017. Extra-corporeal membrane oxygenation for refractory cardiogenic shock after adult cardiac surgery. J Cardiothorac Surg. Jul 17;12(1):55.

Magnussen C, Bernhardt AM, Ojeda FM, et al. 2018. Gender differences and outcomes in left ventricular assist device support: The European Registry for Patients with Mechanical Circulatory Support. J Heart Lung Transplant. Jan;37(1):61-70.

Makrygiannis G, Courtois A, Drion P, Defraigne JO, Kuivaniemi H,
Sakalihasan N. 2014. Sex differences in abdominal aortic aneurysm: the role of sex hormones. Ann Vasc Surg. Nov;28(8):1946-58.

Noah MA, Peek GJ, Finney SJ, et al. 2011. Referral to an extracorporeal membrane oxygenation center and mortality among patients with severe 2009 influenza A(H1N1). JAMA. Oct 19;306(15):1659-68.

Ouweneel DM, Schotborgh JV, Limpens J, et al. 2016. Extracorporeal life support during cardiac arrest and cardiogenic shock. Intensive Care Med. Dec;42(12):1922-1934.

Peek GJ, Elbourne D, Mugford M, et al. 2010. Randomized controlled trial and parallel economic evaluation of conventional ventilatory support versus extracorporeal membrane oxygenation for severe adult respiratory failure (CESAR). Health Technol Assess. Jul;14(35):1-46.

Philipp A, Arlt M, Amann M, et al. 2011. First experience with the ultra compact mobile extracorporeal membrane oxygenation system Cardiohelp in interhospital transport. Interact Cardiovasc Thorac Surg. Jun;12(6):978-81.

Sayer GT, Baker JN, Parks KA. 2012. Heart rescue: the role of mechanical circulatory support in the management of severe refractory cardiogenic shock. Curr Opin Crit Care. Oct;18(5):409-16.

Sheu JJ, Tsai TH, Lee FY, et al. 2010. Early extracorporeal membrane oxygenator-assisted primary percutaneous coronary intervention improved 30-day clinical outcomes in patients with ST-segment elevation myocardial infarction complicated with profound cardiogenic shock. Crit Care Med. Sep;38(9):1810-7.

Thiagarajan RR, Brogan TV, Scheurer MA, Laussen PC, Rycus PT, Bratton SL. 2009. Extracorporeal membrane oxygenation to support cardiopulmonary resuscitation in adults. Ann Thorac Surg. Mar;87(3):778-85. 